\title{
Energy dependence of underlying-event observables from RHIC to LHC energies
}

\author{
Antonio Ortiz $\oplus^{*}$ \\ Instituto de Ciencias Nucleares, Universidad Nacional Autónoma de México, \\ Apartado Postal 70-543, México Distrito Federal 04510, México
}

(Received 20 August 2021; accepted 29 September 2021; published 21 October 2021)

\begin{abstract}
A study of the charged-particle density (number density) in the transverse region of the dihadron correlations exploiting the existing $\mathrm{pp}$ and $\mathrm{p} \overline{\mathrm{p}}$ data from RHIC to $\mathrm{LHC}$ energies is reported. This region has contributions from the underlying event (UE) as well as from initial- and final-state radiation (ISR-FSR). Based on the data, a two-component model is built. This has the functional form $\alpha s^{\alpha}+\beta \log (s)$, where the logarithmic $(\beta=0.140 \pm 0.007)$ and the power-law $(\alpha=0.270 \pm 0.005)$ terms describe the components more sensitive to the ISR-FSR and UE contributions, respectively. The model describes the data from RHIC to LHC energies; the extrapolation to higher energies indicates that at around $\sqrt{s} \approx 100 \mathrm{TeV}$ the number density associated to UE will match that from ISR-FSR. Although this behavior is not predicted by PYTHIA 8.244, the power-law behavior of the UE contribution is consistent with the energy dependence of the parameter that regulates multiparton interactions. Using simulations, KNO-like scaling properties of the multiplicity distributions in the regions sensitive to either UE or ISR-FSR are also discussed. The results presented here can be helpful to constrain QCD-inspired Monte Carlo models at the future circular collider energies, as well as to characterize the UE-based event classifiers which are currently used at the LHC.
\end{abstract}

DOI: $10.1103 /$ PhysRevD.104.076019

\section{INTRODUCTION}

The inelastic proton-proton (pp) cross section has contributions from diffractive (single-diffraction and doublediffraction) and nondiffractive processes. For nondiffractive processes, occasionally, a hard parton-parton scattering occurs producing jets and high transverse momentum $\left(p_{\mathrm{T}}\right)$ particles. The underlying event (UE) consists of particles from the proton breakup (beam-beam remnants) and the multiparton interactions (MPI) that accompany such a hard scattering [1]. Multiparton interactions, i.e., two or more semihard parton-parton scatterings within the same pp collision, are a natural consequence given the composite nature of hadrons [2]. Several data support the presence of MPI in hadronic interactions [3-17]. The successful description of pp collisions by Monte Carlo (MC) generators relies on the precise modeling of UE [2].

The study of pp collisions is also important given the discovery of heavy-ion-like effects in high-multiplicity pp collisions [18]: long-range azimuthal correlations [19], radial flow [20], and strangeness enhancement [21].

\footnotetext{
*antonio.ortiz@nucleares.unam.mx
}

Published by the American Physical Society under the terms of the Creative Commons Attribution 4.0 International license. Further distribution of this work must maintain attribution to the author(s) and the published article's title, journal citation, and DOI. Funded by SCOAP ${ }^{3}$.
In heavy-ion collisions those effects are attributed to the production of a deconfined hot and dense QCD medium, known as the strongly interacting quark-gluon plasma $[22,23]$. Because, e.g., MPI and color reconnection (CR) can produce collective-like effects [24], particle production as a function of quantities sensitive to MPI has attracted the interest of the heavy-ion community [25-29].

Experimentally, inelastic pp collisions are selected using a minimum-bias trigger, while the UE has to be studied in events in which a hard scattering has occurred. This can be achieved by selecting events with a high transverse momentum (e.g., $p_{\mathrm{T}}^{\text {trig }} \geq 5 \mathrm{GeV} / c$ ) charged particle at midpseudorapidity. The activity in the transverse region of the dihadron correlations is the most sensitive to UE, but it also has contributions from initial- and final-state radiation (ISR-FSR). In this paper, the available underlying-event data measured at the Relativistic Heavy Ion Collider (RHIC), the Tevatron, and the Large Hadron Collider (LHC) energies are investigated. The CDF Collaboration subdivided the transverse region into trans-max and trans-min in order to increase the sensitivity to ISR-FSR and UE (beam-beam remnant and MPI) effects, respectively [30]. The UE component was found to increase like a power of the center-of-mass energy, while the ISR-FSR component increased logarithmically. Moreover, according to the MC generators PYTHIA 8.244 [31] and HERWIG 7.2 [32], the $p_{\mathrm{T}}$ spectra and the particle composition are significantly different in the trans-max and 
trans-min regions [33]. The present paper reports a datadriven model based on the UE measurements at RHIC, Tevatron, and LHC energies. Last but not least, the physics opportunities at the Future Circular Collider (FCC) include the effects of the MPI mechanism and its connection with heavy-ion-like phenomena in proton-proton collisions $[34,35]$. Therefore, the data-driven predictions are extended up to $\sqrt{s} \approx 100 \mathrm{TeV}$. The results are compared with the PYTHIA 8.244 MC generator (Monash 2013 tune [36]), hereinafter referred to as PYTHIA 8, for pp collisions at $\sqrt{s}$ from $0.2 \mathrm{TeV}$ up to $100 \mathrm{TeV}$. Finally, in order to improve the understanding of the scaling properties of the multiplicity distributions in the transverse region [37], the studies are extended to higher multiplicities and lower and higher center-of-mass energies, and for the transverse, trans-max, and trans-min regions.

The article is organized as follows: Sec. II provides information about the analysis approach, as well as simulations using the PYTHIA 8 Monte Carlo generator. Section III presents the results and discussion, and finally Sec. IV summarizes the results.

\section{UNDERLYING EVENT OBSERVABLES}

The underlying-event analysis starts from the selection of the highest transverse momentum $\left(p_{\mathrm{T}}^{\text {trig }}\right)$ charged particle of the event. The transverse region is defined by the associated particles within $\frac{\pi}{3}<|\Delta \phi|<\frac{2 \pi}{3}$, where $\Delta \phi$ is the relative azimuthal angle, $\Delta \phi=\phi^{\text {trig }}-\phi^{\text {assoc }}$, with $\phi^{\text {trig }}$ $\left(\phi^{\text {assoc }}\right)$ the azimuthal angle of the trigger (associated) particle [1]. The charged-particle density in the transverse region (number density) is known to rise steeply for low values of $p_{\mathrm{T}}^{\text {trig }}$ and reaches a plateau at $p_{\mathrm{T}}^{\text {trig }} \approx 5 \mathrm{GeV} / c$ (see e.g., Ref. [15]). The selection of events with a high- $p_{T}$ trigger particle biases the sample towards pp collisions with low impact parameter and, hence, high event activity (MPI). UE data include the average multiplicity at the plateau considering only primary charged particles with $p_{\mathrm{T}} \geq 0.5 \mathrm{GeV} / c$. This allows for comparison among different experiments.

Figure 1 shows the number density for various experiments at RHIC [8], Tevatron [30,38], and LHC [9-11,13-15]. Except for the ALICE data point for pp collisions at $13 \mathrm{TeV}$, all the values were taken from the compilation reported by the STAR Collaboration [8]. While the activity shows a modest increase from $\sqrt{s}=0.2$ up to $0.9 \mathrm{TeV}$, for higher energies, it exhibits a steeper rise. The behavior at higher energies is qualitatively similar to the center-of-mass energy dependence of the average number of MPI. The number density is compared with the average charged-particle density (scaled by $2 \pi$ ) measured by ATLAS $[39,40]$ for inelastic pp collisions. As reported by the STAR Collaboration [8], the number density increases faster with the center-of-mass energy than the average multiplicity in inelastic pp collisions. In order to investigate whether this behavior is attributed to UE or

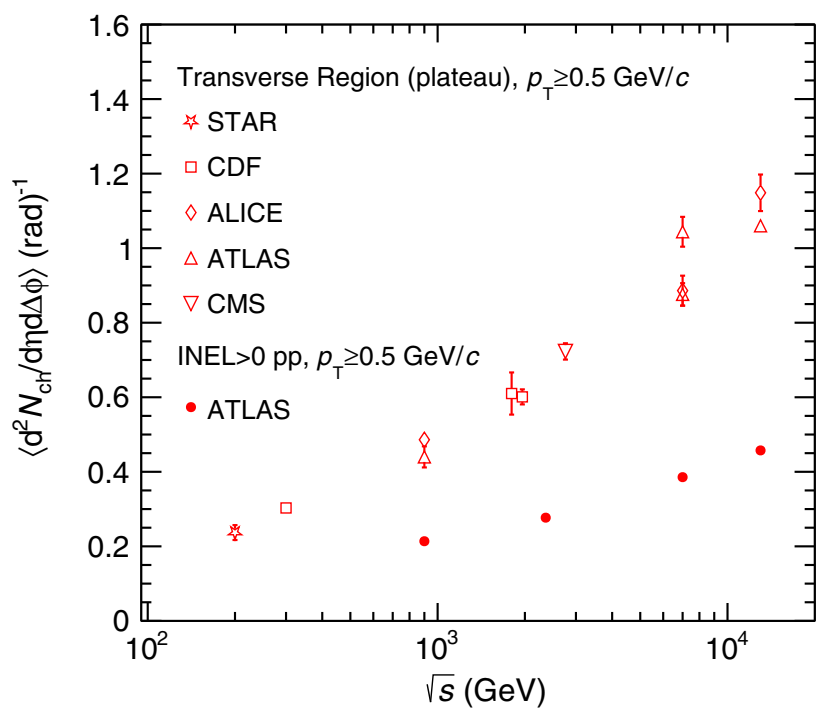

FIG. 1. Center-of-mass energy dependence of the number density reported by various experiments at RHIC, Tevatron, and LHC. Except for the ALICE data at $13 \mathrm{TeV}$, all the values were taken from Ref. [8]. These measurements are compared with $\left\langle\mathrm{d} N_{\mathrm{ch}} / \mathrm{d} \eta\right\rangle$ in INEL $>0$ pp collisions (scaled by $1 / 2 \pi$ ) $[39,40]$. The number densities are obtained by considering final-state charged particles with $p_{\mathrm{T}} \geq 0.5 \mathrm{GeV} / c$. Error bars represent statistical and systematic uncertainties summed in quadrature.

ISR-FSR, a further treatment of the transverse side is implemented.

The transverse region is subdivided into two regions:

(i) transverse-I: $\pi / 3<\Delta \phi<2 \pi / 3$

(ii) transverse-II: $\pi / 3<-\Delta \phi<2 \pi / 3$

The overall transverse region corresponds to combining the transverse-I and transverse-II regions. These two distinct regions are characterized in terms of their relative charged-particle multiplicities. Trans-max (trans-min) refers to the transverse region (I or II) with the largest (smallest) number of charged particles. According to earlier investigations [30,33], these subregions help to separate the ISR-FSR from the UE component of the collision. In the next section, the available trans-max and trans-min data from the CDF experiment will be used to build a model aimed at describing the activity in the transverse region. Results will be compared with PYTHIA 8 . This MC generator is able to describe the underlying-event activity in a hard scattering process, and it was observed earlier that it well describes the measured data by several experiments at the LHC; see e.g., $[9,10,41,42]$. The simulations consist of $5 \times 10^{8}$ inelastic pp collisions for each center-of-mass energy $(\sqrt{s}=0.2,0.9,2.36,7,13,50$, and $100 \mathrm{TeV}$ ). Only final-state charged particles were accepted, excluding the weak decays of strange particles, in order to meet the experimental conditions. Only events with a trigger charged particle with $p_{\mathrm{T}}^{\text {trig }} \geq 5 \mathrm{GeV} / c$ are considered. 


\section{RESULTS AND DISCUSSION}

The CDF Collaboration has measured the number density in the transverse regions for $\mathrm{p} \overline{\mathrm{p}}$ collisions from $\sqrt{s}=0.3$ up to $1.96 \mathrm{TeV}$ [30]. To allow for comparisons with experiments at the LHC, the measurement considered charged particles within $p_{\mathrm{T}} \geq 0.5 \mathrm{GeV} / c$ and $|\eta|<0.8$. The number density for trans-min (more sensitive to MPI) was found to increase much faster with the center-of-mass energy than the trans-max (more sensitive to ISR-FSR). The CDF results are shown in Fig. 2 along with the data discussed in Fig. 1.

The CDF Collaboration reported that trans-dif, i.e., trans-max-trans-min, increases logarithmically with $\sqrt{s}$, while the trans-min increases like a power of the center-ofmass energy. Motivated by this result, a function of the form $s^{\alpha}+\beta \log (s)$ was simultaneously fitted to the existing data on number density in the transverse, trans-min, and trans-max regions, where the terms $s^{\alpha}$ and $\log (s)$ were constrained by the trans-min and trans-max data, respectively. The transverse region was found to be described by such a function with the parameters $\alpha=0.270 \pm 0.005$ and $\beta=0.140 \pm 0.007$. Given that in data the systematic uncertainty is significantly larger than the statistical one, the systematic errors from the data were propagated to the parametrization as follows. Using a random number generator, each data point was shifted up and down within one

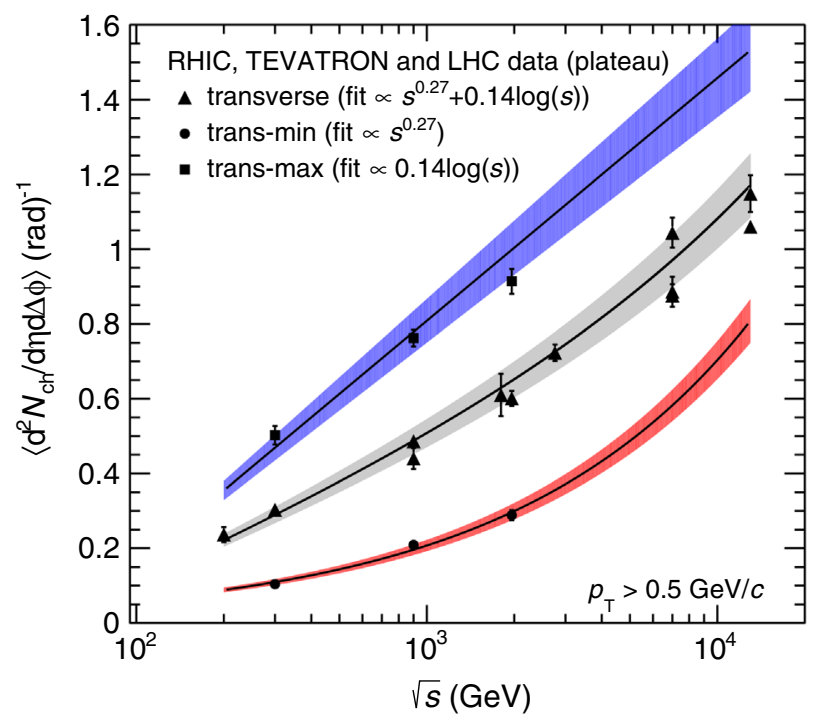

FIG. 2. Center-of-mass energy dependence of the average charged-particle density in the transverse, trans-min, and transmax regions at the plateau in pp [8-11,13-15,30,38] and/or $p \bar{p}$ collisions [30]. The number densities are obtained by considering final-state charged particles with $p_{\mathrm{T}}>0.5 \mathrm{GeV} / c$. The data for the transverse region are compared with a parametrization of the form $s^{\alpha}+\beta \log (s)$, where the first term describes the MPIsensitive region and the second one describes the one more sensitive to ISR-FSR. The shaded areas indicate the one-sigma systematic uncertainty. sigma of the systematic uncertainty. The two-component model was then fitted to the data. The process was repeated 500 times. The sigma of the distribution: (data-fit)/fit was assigned as the systematic uncertainty to the parametrization. It amounts to around $7.2 \%$, and by construction, it is constant as a function of the center-of-mass energy. The determination of the systematic uncertainties of the parameters $\alpha$ and $\beta$ followed a similar procedure. The parametrizations, along with the one-sigma systematic uncertainty, are shown in Fig. 2. Within uncertainties, the number density as a function of $\sqrt{s}$ measured at RHIC, Tevatron, and LHC energies is well described by the two-component data-driven model. It is worth mentioning that only published data are shown in the figure; however, the ATLAS Collaboration has preliminary results for trans-min and trans-max [43]. The preliminary number density for trans-min (trans-max) is around 0.82 (1.34) at $p_{\mathrm{T}}^{\text {trig }}=5 \mathrm{GeV} / c$ for pp collisions at $\sqrt{s}=13 \mathrm{TeV}$, which is slightly above (below) the data-driven prediction. Based on this parametrization, we observe that the activity in trans-min increases faster than trans-max. For example, at $\sqrt{s}=0.9 \mathrm{TeV}$ the activity in trans-max (trans-min) is $\approx 0.78(\approx 0.2)$, whereas the activity at $\sqrt{s}=13 \mathrm{TeV}$ is $\approx 1.53(\approx 0.81)$. This suggests that the MPI contribution increases by a factor $\approx 4$, while that which has a contribution from ISR-FSR increases by about a factor $\approx 2$. Regarding the charged-particle density for inelastic $\mathrm{pp}$ collisions, the increase from $\sqrt{s}=0.9 \mathrm{TeV}$ to $13 \mathrm{TeV}$ is slightly higher than 2 .

In MC generators the energy evolution of MPI is implemented phenomenologically through a transverse momentum cutoff, $p_{\mathrm{T} 0}$, of a few $\mathrm{GeV} / c$. In the original PYTHIA modeling, the energy dependence of the total cross section was taken as the guideline for the energy evolution of $p_{\mathrm{T} 0}$ [2]. Namely, the perturbative MPI cross sections are suppressed below the $p_{\mathrm{T} 0}$ scale, whose evolution with center-of-mass energy is driven by a power law:

$$
p_{\mathrm{T} 0}^{2}(s)=p_{\mathrm{T} 0}^{2}\left(s_{0}\right)\left(\frac{s}{s_{0}}\right)^{b} .
$$

Therefore, a higher scaling power $b$ implies a lower increase of the overall MPI activity. Modern tunes of PYTHIA yield $b$ values in the range $0.21-0.26$ [34]. For example, the Monash tune considers $b=0.215$ and $\sqrt{s_{0}}=$ $7 \mathrm{TeV}$ [36]. The number density as a function of $\sqrt{s}$ in PYTHIA 8 for the three topological regions is displayed in Fig. 3. Results from $\sqrt{s}=0.2 \mathrm{TeV}$ up to $100 \mathrm{TeV}$ considering charged particles within $|\eta|<1$ are displayed. A power-law function describes quite well the MPI-sensitive region (trans-min); the exponent is found to be $\approx 0.23$ which is below that obtained for data and close to the $b$ value which enters in the Monash tune [36]. Contrary to the data-driven prediction, a similar power-law behavior is also observed for the ISR-FSR-sensitive region. In order to 


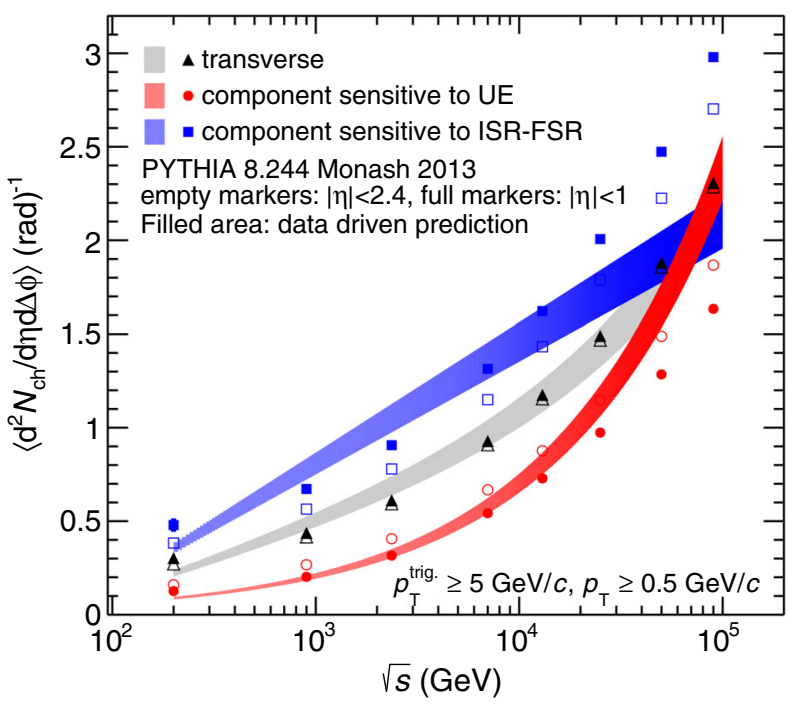

FIG. 3. Center-of-mass energy dependence of the average charged-particle density in the MPI- and ISR-FSR-sensitive topological regions at the plateau $\left(p_{\mathrm{T}}^{\text {trig }} \geq 5 \mathrm{GeV} / c\right)$ in $\mathrm{pp}$ collisions simulated with PYTHIA 8 (tune Monash). Simulations are compared with the extrapolations using data at Tevatron energies (see the text for more details).

investigate the pseudorapidity dependence, PYTHIA 8 predictions are also displayed considering charged particles within $|\eta|<2.4$. While the transverse region is roughly pseudorapidity independent, the number density in transmax (trans-min) exhibits an increase (decrease) with respect to the results considering a narrower pseudorapidity range. This suggests that hard radiation effects increase with the reduction of the pseudorapidity interval used in the analysis. Moreover, the number density increase from the lowest LHC energies $(\sqrt{s}=0.9 \mathrm{TeV})$ up to $100 \mathrm{TeV}$ is around 4.4 (8.2) for the ISR-FSR-sensitive region (MPIsensitive region). The data-driven model predicts that the increase of the activity in the MPI-sensitive region should be around 12 going from $\sqrt{s}=0.9 \mathrm{TeV}$ up to $100 \mathrm{TeV}$, whereas the increase for the ISR-FSR-sensitive region is around 2.7, which is smaller than predicted by PYTHIA 8 . The discrepancy at FCC energies could be due to the tuning of soft and semihard physics in Monte Carlo event generators, which relies on parton distribution functions (PDFs) in unexplored kinematical regions (e.g., $x \lesssim 10^{-5}$ ). Therefore, the data-driven model could be useful to improve the MC predictions at FCC energies.

In the analysis of data, the relative contribution from MPI with respect to that from ISR-FSR is expected to play a role in observables like $p_{\mathrm{T}}$ spectra of unidentified charged particles, as well as in particle ratios like $(\mathrm{p}+\overline{\mathrm{p}}) /\left(\pi^{+}+\pi^{-}\right)$as a function of $p_{\mathrm{T}}$. If radiation plays an important role, then the particle ratios will be significantly suppressed with the increase of the event multiplicity on the transverse side. On the other hand, if the MPI component is the dominant contribution, then the particle ratios will be significantly enhanced at intermediate $p_{\mathrm{T}}$ with increased event activity on the transverse side. This has been reported in Ref. [33], where the features of particle ratios as well as $p_{\mathrm{T}}$ spectra as a function of the activity in transverse, trans-max, and trans-min regions were

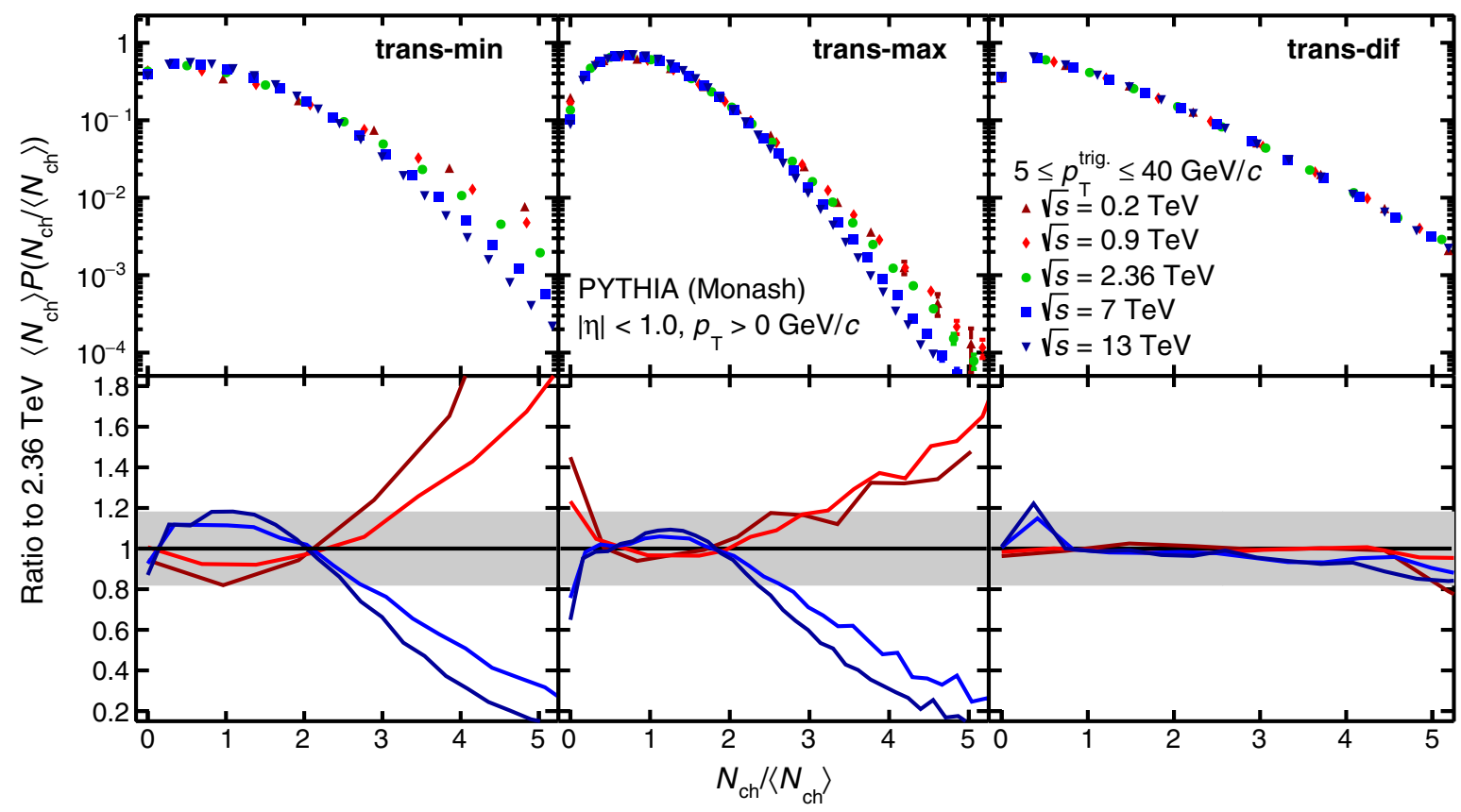

FIG. 4. Top: charged-particles multiplicity distributions in KNO variables in pp collisions simulated with PYTHIA 8. Results for different center-of-mass energies are shown for the trans-min (left), trans-max (middle), and trans-dif (right) regions. Bottom: KNO multiplicity distributions normalized to that for pp collisions at $\sqrt{s}=2.36 \mathrm{TeV}$. 
investigated. The preliminary ALICE data [28] suggest that the relative contribution from ISR-FSR with respect to that from MPI is smaller in data than in PYTHIA 8 [33]. Early LHC data already suggested that the MPI activity at the LHC energies was higher than in PYTHIA [44].

Last but not least, it has been reported that, within $20 \%$, the multiplicity distributions in the transverse region $\left(|\eta|<2.5, p_{\mathrm{T}}>0 \mathrm{GeV} / c\right)$ at the plateau obey a KobaNielsen-Olesen (KNO) scaling [37]. This scaling was expected in a model which assumes that a single pp collision results from the superposition of a given number of elementary partonic collisions emitting independently [45]. Therefore, MPI could produce such an effect. In [37], it was shown that the scaling held for pp collisions at the LHC energies for $0.5<z\left(=N_{\mathrm{ch}} /\left\langle N_{\mathrm{ch}}\right\rangle\right)<2.5$. Now, a refinement of that result is reported. The primary goal is to investigate the KNO-like scaling properties if the sensitivity to MPI is improved. The impact of radiation is investigated using the multiplicity distributions in transmax, as well as trans-dif. PYTHIA 8 results are reported for pp collisions from RHIC to LHC energies and for the pseudorapidity interval $|\eta|<1$, which allows us to extend the $z$ reach. Figure 4 shows the multiplicity distributions in $\mathrm{KNO}$ variables for pp collisions at $\sqrt{s}=0.2,0.9,2.36,7$, and $13 \mathrm{TeV}$. The results for trans-max are qualitatively similar to those reported for the transverse region [37]. Namely, the KNO-like scaling holds for $0.5<z<2.5$, whereas for lower or higher values of $z$, the violation of the $\mathrm{KNO}$-like scaling is bigger than $20 \%$. It is worth noticing that for trans-max, both contributions are considered: UE and ISR-FSR. If the effect of ISR-FSR is suppressed, i.e., exploiting the features of the trans-min region, then the $\mathrm{KNO}$-like scaling is extended up to very low multiplicities $(z<0.5)$, whereas for $z>2.5$ the KNO-like scaling is still broken. Events with high multiplicity jets can contribute to the violation of the scaling properties. For example, a quantity sensitive to the number of MPI as a function of the event multiplicity is presented in Refs. [12,17]. It was observed that for $z>3$, the number of uncorrelated seeds (or MPI) deviates from the linear trend, suggesting the presence of high multiplicity jets. Finally, results for trans-dif show a perfect KNO scaling in a broader $z$ interval, i.e., from 0 up to 5. This result complements the finding reported in Ref. [37], suggesting that the hardest component of UE exhibits perfect scaling properties, whereas the MPI gives an approximate scaling which holds up to $z \approx 2.5$.

\section{CONCLUSIONS}

This article reports a data-driven model which is built using the existing UE data from RHIC up to LHC energies. The function which describes the data is of the form $\propto s^{0.27}+0.14 \log (s)$, where the power-law term $(\alpha=0.270 \pm 0.005)$ and the logarithmic term $(\beta=0.140 \pm 0.007)$ describe the MPI- and ISR-FSRsensitive topological regions of the collision, respectively. Albeit in PYTHIA, the MPI-sensitive region is also well described by a power-law function $\left(\propto s^{0.23}\right)$, such a contribution is found to increase faster with $\sqrt{s}$ in the datadriven model than in PYTHIA 8.244. It is worth mentioning that the exponent which was found for PYTHIA 8 is close to that which enters in the Monash tune for the parametrization of the energy dependence of MPI. This paper also reports that at the FCC energies, the MPI contribution is expected to dominate the transverse region, whereas the opposite behavior is predicted by PYTHIA 8.244. One has to consider that the MC prediction relies on parton distribution functions in the ultra-low- $x$ regimen which has not been explored so far. Finally, the multiplicity distributions for each region were investigated considering pp collisions simulated with PYTHIA 8.244 from $\sqrt{s}=0.2$ up to $13 \mathrm{TeV}$. A KNO-like scaling is predicted for the MPI-sensitive region; it would hold from $z=0$ up to $z=2.5$. For higher $z$ values, high multiplicity jets may break the scaling properties. The KNO scaling is broken at low $z$ values $(z<0.5)$ when radiation is folded together with the MPI contribution. However, when ISR-FSR effects are fully isolated, the scaling holds for a wide $z$ interval, from 0 up to 5. Data for trans-min and trans-max at the LHC energies would be needed in order to check the validity of the proposed parametrizations and the scaling properties.

\section{ACKNOWLEDGMENTS}

The author acknowledges very useful discussions with Guy Paić and Peter Christiansen. Support for this work has been received from CONACyT under Grant No. A1S-22917.
[1] R. Field, Annu. Rev. Nucl. Part. Sci. 62, 453 (2012).

[2] T. Sjöstrand and M. van Zijl, Phys. Rev. D 36, 2019 (1987).

[3] T. Åkesson et al. (Axial Field Spectrometer Collaboration), Z. Phys. C 34, 163 (1987).

[4] F. Abe et al. (CDF Collaboration), Phys. Rev. D 56, 3811 (1997).
[5] V. M. Abazov et al. (D0 Collaboration), Phys. Rev. D 81, 052012 (2010).

[6] D. Acosta et al. (CDF Collaboration), Phys. Rev. D 70, 072002 (2004).

[7] T. Aaltonen et al. (CDF Collaboration), Phys. Rev. D 82, 034001 (2010). 
[8] J. Adam et al. (STAR Collaboration), Phys. Rev. D 101, 052004 (2020).

[9] G. Aad et al. (ATLAS Collaboration), Phys. Rev. D 83, 112001 (2011).

[10] B. Abelev et al. (ALICE Collaboration), J. High Energy Phys. 07 (2012) 116.

[11] G. Aad et al. (ATLAS Collaboration), Eur. Phys. J. C 74, 2965 (2014),

[12] B. Abelev et al. (ALICE Collaboration), J. High Energy Phys. 09 (2013) 049.

[13] V. Khachatryan et al. (CMS Collaboration), J. High Energy Phys. 09 (2015) 137.

[14] M. Aaboud et al. (ATLAS Collaboration), J. High Energy Phys. 03 (2017) 157.

[15] S. Acharya et al. (ALICE Collaboration), J. High Energy Phys. 04 (2020) 192.

[16] A. Ortiz, A. Paz, J. D. Romo, S. Tripathy, E. A. Zepeda, and I. Bautista, Phys. Rev. D 102, 076014 (2020).

[17] A. Ortiz and E. A. Zepeda, J. Phys. G 48, 085014 (2021).

[18] J. L. Nagle and W. A. Zajc, Annu. Rev. Nucl. Part. Sci. 68, 211 (2018).

[19] V. Khachatryan et al. (CMS Collaboration), Phys. Lett. B 765, 193 (2017).

[20] S. Acharya et al. (ALICE Collaboration), Phys. Rev. C 99, 024906 (2019).

[21] J. Adam et al. (ALICE Collaboration), Nat. Phys. 13, 535 (2017).

[22] R. Bala, I. Bautista, J. Bielcikova, and A. Ortiz, Int. J. Mod. Phys. E 25, 1642006 (2016).

[23] W. Busza, K. Rajagopal, and W. van der Schee, Annu. Rev. Nucl. Part. Sci. 68, 339 (2018).

[24] A. Ortiz, P. Christiansen, E. Flores, I. Cervantes, and G. Paic, Phys. Rev. Lett. 111, 042001 (2013).

[25] T. Martin, P. Skands, and S. Farrington, Eur. Phys. J. C 76, 299 (2016).

[26] A. Ortiz and L. Valencia Palomo, Phys. Rev. D 99, 034027 (2019).

[27] V. Zaccolo (ALICE Collaboration), Springer Proc. Phys. 250, 245 (2020).
[28] A. Nassirpour (ALICE Collaboration), J. Phys. Conf. Ser. 1602, 012007 (2020).

[29] G. Bencédi, A. Ortiz, and S. Tripathy, J. Phys. G 48, 015007 (2021).

[30] T. A. Aaltonen et al. (CDF Collaboration), Phys. Rev. D 92, 092009 (2015).

[31] T. Sjöstrand, S. Ask, J. R. Christiansen, R. Corke, N. Desai, P. Ilten, S. Mrenna, S. Prestel, C. O. Rasmussen, and P. Z. Skands, Comput. Phys. Commun. 191, 159 (2015).

[32] J. Bellm, S. Gieseke, D. Grellscheid, P. Kirchgaeer, F. Loshaj, G. Nail, A. Papaefstathiou, S. Pltzer, R. Podskubka, M. Rauch, C. Reuschle, P. Richardson, P. Schichtel, M. H. Seymour, A. Sidmok, and S. Webster, arXiv:1705.06919.

[33] G. Bencedi, A. Ortiz, and A. Paz, Phys. Rev. D 104, 016017 (2021).

[34] M. Mangano et al., CERN Report No. CERN-2017-003-M, 2017, https://cds.cern.ch/record/2270978.

[35] A. Abada et al. (FCC Collaboration), Eur. Phys. J. ST 228, 755 (2019).

[36] P. Skands, S. Carrazza, and J. Rojo, Eur. Phys. J. C 74, 3024 (2014).

[37] A. Ortiz and L. Valencia Palomo, Phys. Rev. D 96, 114019 (2017).

[38] T. Affolder et al. (CDF Collaboration), Phys. Rev. D 65, 092002 (2002).

[39] G. Aad et al. (ATLAS Collaboration), New J. Phys. 13, 053033 (2011).

[40] G. Aad et al. (ATLAS Collaboration), Phys. Lett. B 758, 67 (2016).

[41] V. Khachatryan et al. (CMS Collaboration), Eur. Phys. J. C 70, 555 (2010).

[42] S. Chatrchyan et al. (CMS Collaboration), J. High Energy Phys. 09 (2011) 109.

[43] S. Tokar, in Proceedings of the 17th Conference on Elastic and Diffractive Scattering (2017) [arXiv:1711.01180].

[44] B. Abelev et al. (ALICE Collaboration), Eur. Phys. J. C 72, 2124 (2012).

[45] J. Dias de Deus, C. Pajares, and C. A. Salgado, Phys. Lett. B 408, 417 (1997). 